\title{
Application of a physical science model in the analysis of patient flow in a hospital
}

\author{
Sinval Lins Silva*1, José M.A. Figueiredo² \\ ${ }^{1}$ Núcleo de Ciências da Saúde, Hospital Felicio Rocho, Belo Horizonte, Minas Gerais, Brazil \\ ${ }^{2}$ Departamento de Física, Universidade Federal de Minas Gerais, UFMG, Belo Horizonte, Minas Gerais, Brazil
}

Received: November 14, 2018

DOI: $10.5430 /$ jha.v8n1p34
Accepted: December 28, 2018 Online Published: January 2, 2019

URL: https://doi.org/10.5430/jha.v8n1p34

\begin{abstract}
One of the most relevant aspects in hospital management relies on how to properly control and predict the patient flow, that is, the paths and the time sequence a whole set of patients run in their journey inside the hospital, as they look for treatment. This issue is of the utmost importance since it interferes in the quality of the healthcare delivered to a person and also has a huge impact on both the costs for the patient and the operational costs for the hospital. This work intends to collaborate with the comprehension of the patient flow analysis and to offer a mathematical model analogous to a physical science model capable of, qualitatively at first sight, describing the main variables and properties of this flow. We also present the logical elements that allow the manager to develop quantitative flow evaluations adaptable to a specific institution, based on local measurements of the variables described here. This theoretical formulation can be applied directly to practical situations concerning the management of patient flow. The relevant variables and their mathematical relations can be used by the manager in order to quantify each relevant patient circuit in a hospital. This way, it is expected that recurring problems derived from the unwanted variations in the patient flow can be anticipated and corrected by the manager.
\end{abstract}

Key Words: Patient flow, Healthcare improvement, Ohm's law, Hospital management, Operations management

\section{INTRODUCTION}

Packed services, delayed treatments, evasion and attendance in hallways often plague emergency services in hospitals. These annoying effects derived from saturated services show how complex the interaction of the variables that rule patient flow are and challenge healthcare institutions all around the world. Patient flow, though notorious in emergency departments, is a systemic problem in all areas of a hospital. The interaction between intensive care units, operating rooms, patient care units and emergency departments must be analyzed as a whole. ${ }^{[1-4]}$ In this scenario, managing patient care has attracted the attention of researchers and healthcare professionals, who have sought to formalize and model this management. ${ }^{[1-23]}$

In this work we present a model analogous to certain models that describe well-established physical phenomena and when applied to the management of patient flow in hospitals, can help the administrator to solve the issues related to it. The analogy we have made here has the purpose to serve as a guide to the manager in the idealization of actions that contribute to the solution of the issues mentioned here. We also demonstrate how the existing solution tools for managing the patient flow relate among themselves.

In the following sections, we used the equations appropriated

\footnotetext{
*Correspondence: Sinval Lins Silva; Email: sinval.lins@ gmail.com; Address: Avenida do Contorno 9530, Belo Horizonte, Minas Gerais, 30.110-934,
} Brazil. 
to an electrical circuit in order to model a circuit for the patient flow. This formulation has the advantage of including the conceptual and operational elements that are relevant to this problem and thus allowing the rational use of these elements in the search for specific solutions in a hospital service. Finally, we analyzed how the patient flow can depend on the characteristic parameters of a hospital, and proposed a new interpretation for the Theory of Constraints based on the Continuity Equation (a theory that explains mass transport in a constricted region, such as a channel, for instance).

\section{Methodology}

\subsection{A physical approach to the patient flow problem}

When patients look for a hospital, they go through attendance lines that form a circuit, from their entry to their exit. This patient circuit can be compared to both the conductors in series and in parallel, as in an electrical circuit. Such conductors form a general circuit, which can be the hospital as a whole or a specific unit, such as the emergency department, the surgical ward, the laboratory, the intensive care unit or the patient care unit. A circuit can also be understood as the treatment to patients with a specific illness or as any administrative process, for instance, the billing system. In this way, each general attendance circuit is formed by specific intermediate circuits of receptionists, triage, physicians, nurses, image technicians and their equipment, laboratory technicians and their equipment, administrative personnel, beds and available chairs. Each element of a specific intermediate circuit is a local circuit, which in turn is a conductor that can no longer be subdivided.

In the context of this work and in analogy with the physical models, we introduce the following elements of the circuit of patient flow, with the goal of elaborating a specific analog model:

a) Entry Point: place where the patients enter to be treated, either coming from home or from another attendance circuit.

b) Exit Point: place where patients leave to go home or to another circuit. The physical distance that the patients go through between the entrance and the exit is one of the determining factors of length $\mathrm{L}$ of the circuit.

c) Patients: are the elements that flow through the circuit and can be compared to a fluid or to electric charges of an electric circuit.

d) Conductors: are the elements that allow the patient flow. They could be physical in nature, such as hallways, the reception area, beds, chairs, imaging and laboratory equipment. For instance, a chair or the space for image services, within the hospital environment, represents a pathway that allows the patient flow and therefore can be considered conductors. On the other hand, the medical team, the nurses, the radiology and laboratory technicians, as well as the administrative personnel can be considered conductors of human nature since the patient flow also happens through them.

In the context of this work and taking under consideration the analogy with the physical models, we introduce the following magnitudes for a patient flow circuit:

a) Pressure $P$ : difference in demand exerted by the number of patients in the circuit's entrance and exit.

b) Flow $\phi$ : the movement of patients within a circuit, being measured by the number of patients that are treated in a time interval " $t$ " through a transversal section of a conductor. We can imagine the circuits as channels, through which the patient flows during the stages of treatment. The transversal section of these channels is an important parameter for the flow.

c) Resistance $R$ : resistance offered by circuit conductors to the patient flow.

d) Internal Friction $\mu$ : magnitude that is intrinsic to each service, inversely proportional to the quality and to the organization of the offered treatment. It reflects the physical, professional and emotional interaction between the actors in the attendance process, which are the patients, the healthcare professionals and the administrative personnel.

e) Length $L$ : sum of the lines of the attendance that are offered to the patient. This length can be physical, associated to the distances the patient goes through within the hospital, or can be technical, linked, in this case, to the duration of the processes the patient is submitted to. Long processes increase the circuit's effective length, even though the patient can remain in the same place.

f) Area $A$ : area of the transversal section of the conductors through which the patient flow $\phi$ happens. This parameter has a physical characteristic, dependent on the hospital's architecture, as well as a human characteristic. The human resource grid available for the attendance interferes decisively in the flow and defines an effective area as well. For instance, there could be an ample reception room for the patient who accesses the hospital, but having an insufficient number of personnel for triage, and this effect acts as a limiting factor for the flow. In this way, it can be said that the inadequate dimensioning of the human resources diminishes the effective area.

\subsection{An empirical model for the patient flow}

When analyzing the tools of management and solution of issues found in patient flow, it was observed that many of 
them are related to conceptual elements that refer to architecture, ${ }^{[1,3]}$ circuits, ${ }^{[9]}$ queues, ${ }^{[14,15]}$ variability, ${ }^{[7]}$ proximity of services to the place of treatment, ${ }^{[9]}$ adjustment of capacity to the demand, ${ }^{[6,9]}$ speed, ${ }^{[5,17]}$ pressure, availability of conductors and attendance lines, parallelism, ${ }^{[10,12,21]}$ organization and quality, standardization and shortening of processes, ${ }^{[20]}$ work sequence, elimination of bottlenecks, ${ }^{[16,17]}$ real time monitoring ${ }^{[3,10,11]}$ and patient triage. Therefore, we propose an analogue physical model for the patient circuit, which relates to several of the terms listed above.

\subsubsection{General equation}

For the patient flow, we propose the following equation to relate the pressure $P$ of patients with their flow $\phi$ (see Equation 1):

$$
P=R \phi
$$

In this equation, $R$ is the resistance of the portion of the circuit that is under analysis. Equation 1 relates a cause $(P$, the difference in pressure of patients between the entrance and the exit of a circuit or a portion of a circuit) with an effect ( $\phi$, the patient flow in this circuit). We also assume, as in the first approximation, that resistance $R$ is constant, meaning that this parameter is independent of the value of the difference in pressure. This approximation is valid for not very high pressure values. In this way compared to the analogy in physics, it should be expected that the value of this parameter depends on geometric factors (architecture) of the circuit. For instance, the increase in the attendance circuit length increases its resistance. On the other hand, wider hallways and ampler rooms can facilitate the treatment access and diminish the resistance of this process. Besides the influence of the geometric effects, the effects of the interaction of the patient with the structure of attendance also interfere with the value of its resistance. In this work the effect of the patient's interaction with the attendance structure is described by friction $\mu$ even though this parameter is difficult to measure in the context of the management tools. We therefore have the following equation (see Equation 2):

$$
R=\frac{\mu L}{A}
$$

Combining Equations 1 and 2, we have the following general equation relating pressure and patient flow (see Equation 3):

$$
P=\frac{\mu L}{A} \phi
$$

That is analogous to Ohm's Law applicable to the patient flow circuit. This equation can be applied to parts of the circuit or to the circuit as a whole.
It is important therefore to establish an important difference in this analogy. In an electrical circuit, resistance always has a deleterious dissipative effect. It transforms the electric energy into heat, which is lost irreversibly in the circuit. In the case of a patient circuit, part of the friction refers to losses (also irreversible) due to the inefficiency of the attendance circuit but here friction is also associated to the interactions of the patients with the service they are seeking in the hospital (consultations, examinations, procedures). From the manager's point of view, even this non-dissipative effect has to be included in the calculation or the measure of the resistance, because at a certain point of the attendance line, the patients (alive or dead) will have to leave the hospital or the circuit under analysis, which alters its pressure's value. At this point of the analysis made here, another aspect of the physical analogy should also be considered. Both the electric current and the patient flow represent average values of the flow of multiple electric charges (from a physics point of view) or of multiple patients, in a hospital. That is to say, patient flow values must be considered as the average of a multiple occurrence of single patient events.

\subsubsection{Analysis of area $A$}

Area $A$ is the transversal section area through which patient flow $\phi$ happens. The patients flow through conductors, whose transversal sections have a rectangular area that is equal to the product of its height $h$ versus its base $b(N)$. The equation is given by (see Equation 4):

$$
A=b(N) h
$$

As the height $h$ of floors of hospital buildings is not a variable, the only parameter that can be modified in area $A$ is its base $b(N)$, where $N$ corresponds to the number of attendance lines available in parallel. Thus, base $b(N)$ is variable and it is a function both of the geometric area and of the available human and technical resources. Therefore, the number $N$ includes the consultation offices, exam rooms, service desks, physicians, nurses, technicians, etc. The bigger the number $N$, the bigger the value of base $b(N)$ and the value of area $A$. Busy attendance lines, or few of them in parallel, reduce the value of $b(N)$ and, consequently, of area $A$.

\subsubsection{Configuration of healthcare delivery system}

Given the elements of the analogy presented here, it must be pointed out that in a circuit of patients the conductors can be in series, in parallel, or in both configurations. In order to avoid the duplication of tasks, the parts of the attendance lines that contain similar local conductors (that form specific intermediate circuits) are habitually set in parallel. On the other hand, the parts that contain different local conductors are habitually set in series (both configurations are similar 
to the electric circuit and to the human circulatory system). The reason for the configuration in series is associated with the need of achieving sequential tasks and procedures, due to either technical or logistical reasons. ${ }^{[24]}$ However, there are tasks of different local conductors that can and should be done in parallel, as will be seen in the following sections.

Therefore, the equations that apply to the calculation of equivalent resistances in an electric circuit are also applied to the calculation of the equivalent resistances of the association of conductors in a circuit of patients. On the other hand, a real circuit of patients is extremely complex because it has several connections between the different services provided, the healthcare professionals and their decision-making process, the patients and their illnesses and their response to treatment. This grid presents countless combinations of conductors, sometimes difficult to map out due to the unpredictability and variability of the processes. ${ }^{[5-9,24]}$ In this way, the manager must be mindful when using the physical analogies in a manner to make it compatible and applicable to the model presented here.

Considering the hospital as a whole, its circuits could have, ideally, the same number of some specific intermediate circuits, in a way that several attendance lines were also available in parallel. For example, the same number of physicians at work, consultation rooms and the associated nursing services.

But an ideal hospital is not a real hospital. ${ }^{[7]}$ In a hospital, the patient is, at the same time, a user and an actor of the production process. This conjunction demands for the existence of an installed work capacity which cannot be stocked to be used afterwards. If this capacity becomes idle, it will be wasted. If a specified service is not used, its availability will be definitively lost. ${ }^{[5]}$ That is why there must be a balance of the following aspects of the process of patient flow: installed capacity and demand, cost and benefit, quality and result, provided service and available area, budget and billing. Besides, it must be pointed out that the patient treatment cannot be based only on statistical data and on average values. ${ }^{[14,18,19]}$ In this context, the medical consultation becomes a complex procedure, and care must be taken in the analysis of its execution time. ${ }^{[25,26]}$ According to Jensen et al., ${ }^{[5] ~ " P a t i e n t ~ v e l o c i t y ~ s h o u l d ~ b e ~ b a l a n c e d ~ w i t h ~ o t h e r ~ a s p e c t s ~}$ of care, like patient satisfaction, quality measures, patient safety, and risk reduction."

\section{RESULTS}

In the sequence, we present a general analysis of the patient flow from the standpoint of the proposed Equation 1.

Published by Sciedu Press

\subsection{General analysis}

It is obvious that electrical circuits do not have in their intimacy anything to do with the flow of people. Besides, Ohm's Law is a linear equation, in which there is a direct relation between tension $V$ and current I. In the patient circuit, a direct relation of proportionality between $P$ and flow $\phi$ can be verified during a great part of the occupation of the installed capacity, for a given increase in the value of $P$. Under this condition, resistance $R$ is nearly constant (that is, it depends very little on the value of difference in pressure $P$ ). It has been observed that the average time spent in a clinic ${ }^{[5,24]}$ and the average delay for attendance ${ }^{[8]}$ remain approximately stable, even when there is an increase in the occupation rate. This means that, even if there is an increase in the pressure of patients, the flow also increases proportionally, because the average time and the average delay are subject to small variations. So the resistance of the circuit does not depend (or weakly depends) on the value of $P$. It has been established that this linear regime could be sustainable at values up to $80 \%$ of the occupation capacity.

In fact, if this situation of direct relation of proportionality were true during the entire time a patient seeks for the hospital, the flow $\phi$ would increase without limit, in response to any increase in $P$. However, this effect does not happen in real life situation. As the value of $P$ increases (due to spontaneous demand from the patients that seek the hospital) and reaches a value that is incompatible with the installed capacity, the saturation of attendance lines is verified. This nonlinear effect generates an increase in resistance $R$ and consequent drop in flow. From this moment resistance $R$ - as presented in Equation 2 - stops being constant and becomes dynamic (meaning it starts to depend on $P$ ). The linearity between $P$ and flow is thus lost. This effect results in an increase of the complexity for the solution of the process and usually demands the inclusion of new analysis to the model.

Lack of linearity is one of the fundamental aspects of the study of the Queuing Theory. ${ }^{[5-9]}$ The biggest evidence of the loss of linearity between $P$ and flow is the increase of the average time and the average delay due to an increase of the occupation rate of the installed capacity. As stated above, even when an occupation rate of $80 \%$ to $85 \%$ of the installed capacity is attained, the average time in clinic ${ }^{[5,24]}$ and the average delay for attendance ${ }^{[8]}$ present small variations, meaning that the capacity absorbs the demand. Above this value range, the average time and the average delay begin to grow exponentially and the saturation of the circuit takes place. Under these conditions, magnitude variations of the parameters in a circuit cannot reflect in a way that is proportional to the flow. For instance, an increase in the area may not have the desired effect in the flow but could 
considerably increase the implementation costs. Therefore, there must be predictability, in order to anticipate and avoid the system reaching the saturation state. It has been observed that the exact place (elbow) of the loss of stability of the average delay depends on two characteristics of the system: variability and its size. According to Hall: ${ }^{[8]}$

"The higher the degree of variability in the system, the more to the left the elbow will be so that delays will be worse for the same utilization level (the greater the variability in the service time, the longer the delays at a given utilization level). And the larger the system, the closer the elbow will be to $100 \%$ so that delays will be smaller for the same utilization level. Unless average utilization is strictly less than $100 \%$, the system will be unstable and the queue continues to grow. Queuing systems have economies of scale so that larger hospitals can operate at higher utilization levels than smaller ones yet maintain similar levels of congestion and delays."

Although there is a consensus among several authors ${ }^{[5-8]}$ with regard to the classification of variability as natural or artificial (as will be seen further ahead), any variation in the values of pressure, internal friction, length and area that increases the resistance and promotes saturation must be discouraged. On the other hand, a variation in these values that decreases the resistance and avoids saturation must be encouraged.

In the following section, we demonstrate how the equation proposed for patient flow explains the saturation of this circuit, considered to be an essential aspect for the management of healthcare units.

\subsection{Saturation of the circuit}

The saturation in the patient flow circuit takes place due to the following factors:

a) The absence of optimization of demands from patients in the entrance of the circuit (for instance, if there is a badly done triage) and in its exit (for instance, lack of agility in discharge) alters the difference in pressure and more attendance lines are occupied, generating a drop in the value of $b(N)$, until the saturation of the circuit. In this case, there would be no available lines left. Thus, area $A$ decreases and resistance $R$ increases in the same proportion. Therefore, a decrease of the flow takes place and followed by the saturation of the circuit. For instance, consider the increase in the prevalence of chronic diseases in the population such as diabetes mellitus and systemic arterial hypertension, among others. These cases increase the demand for emergency room services, even when there is not an indication for such treatment. ${ }^{[27]}$ In this way, the lack of access to primary healthcare units leads to the search for rapid treatment, without necessarily being a medical urgency or emergency. These factors contribute in a significant way to the oscillation in patient pressure in a general hospital. ${ }^{[2,27]}$ One way of reducing the variations in the value of $P$ is to increase the offer of medical attention outside the hospital environment (in the form of annexes) for the patients that need ambulatory services.

b) Services that do not use tools that improve the quality, the organization and the internal communication tend to be disorganized, slow in decision making and full of conflicts between the parts, leading to the increase in internal friction $\mu$. Minimizing the internal friction involves improving all levels of the interaction among the actors of the process, which includes the patients, the healthcare professionals and the administrative personnel.

Oftentimes a pleasant and comfortable environment with air conditioning and clean restrooms exert a reduction in the stress of patients and employees and a decrease in internal friction. The measurement of this magnitude can be made by the tools of the institutions of accreditation and certification, such as ONA, ${ }^{[28]}$ NIAHO, ${ }^{[29]} \mathrm{JCI}^{[30]}$ and ISO.$^{[31]}$ Furthermore, if the resistance of a determined circuit presents itself as being high, but this circuit possesses adequate length and area, the only magnitude that can be adjusted is the internal friction. An elevated internal friction increases the resistance and this is one of the factors that, if not adjusted, could saturate the circuit.

c) An inadequately made architectural planning, with essential services such as image services and laboratories far from the place of treatment, increases the value of $L$. Another common error in the management of patient flow is to increase the value of $L$ by adding a local circuit in series to correct another local circuit whose performance is not satisfactory. The correct way is to improve the deficient local circuit instead of adding another. Long tasks tend to saturate the circuit more quickly. If the patient is obligated to have an exam done in a place that is distant from where she/he is being treated and then returns to that place, this effect increases the traffic time and decreases the flow: the resistance increases. The same could happen if the patient is retained for a long time, e.g. waiting for the result of an exam in a local line. As in the former example, there would be an increase in the traffic time. This increase of the resistance can be analyzed as a consequence of the increase of the length. Besides, unnecessarily long or wasteful processes also increase the length of attendance lines, which make the flow slower, and more time consuming.

d) Each conductor that cannot be subdivided characterizes a local circuit with $N=1$. For local circuits in the parallel 
configuration, if one is not properly working, the others suppress the flow. But for certain patients, an in-series circuit is indispensable and it can be the only element of a specific intermediate circuit. This circuit becomes critical because, if it does not work, there is no flow.

e) Another issue that is important to consider is the area of the attendance circuits. The best way to increase area $A$ is to make available more attendance lines in parallel, which is the best configuration to increase flow $\phi$. However, certain implementation costs could be incompatible with the financial condition of the institution and usually demand long term planning.

But there is another way of increasing the area and here it is important to note that local circuits are characterized by one unique line with $N=1$. Beyond the geometric interpretation, there is also the functional interpretation of the conductor: the availability or receptivity for the task. Local conductors, such as employees with total availability, effectively have their area full and ready for attendance, whereas employees that are slow and dispersed by other matters (cell phones, for instance), with grudges and badly trained, not only make their tasks longer and with more internal friction, but also effectively decrease their area of interaction with the patient ( $N$ ends up having a value between 0 and 1), all of which contributes to the increase of the resistance and saturation of the system.

f) The variability of the flow ${ }^{[5-8,24]}$ could be due to natural processes, such as randomness in the arrival of patients, differences between patients and their illnesses and in the experience of each professional. Such variability cannot be eliminated, but it can be managed. On the contrary, artificial or unnatura ${ }^{[23]}$ variability such as peaks of pressure of elective surgeries in the entrance of the surgical ward, is controllable and could be eliminated. This means, for instance, actions that adequate the number of surgeries to the capacity of the surgical ward. Another artificial variability found is the patient discharge. In nearly $80 \%$ of the time the physicians know with one day's notice when they will discharge the patient. ${ }^{[2,9]}$ Such information is not explored enough, and it is very common to have an unnecessary waiting period for the discharge. ${ }^{[9]}$ In Felicio Rocho Hospital, a pre-discharge protocol was instituted. When the physician makes the summary of the hospitalization and the homecare prescription on the day previous to the discharge, an e-mail is sent to several sectors (such as nursing, billing, bed management, hospitality, laboratory and image department). The process, previously done in a sequential manner, is now done in parallel. This way, permanence of the patients was reduced in two hours. ${ }^{[32]}$

Published by Sciedu Press
Besides the ability to manage, the variability could be used as a source of knowledge. ${ }^{[33]}$ In complex systems, such as a hospital, the processes occur in varied conductors and they present several connections. That is why studying the variations can create opportunities for the conception of new circuits. As a result of internal studies ${ }^{[32]}$ carried out in Hospital Felicio Rocho, it was observed that there was a high demand for certain groups of patients, who oftentimes stayed in lines in the emergency department. The solution to this problem was the creation of specialized treatment services, such as a center for wounds secondary to peripheral vascular insufficiency, an orthopedic clinic, a surgical ward for small surgeries and a center of ambulatory infusion of drugs. Specific attendance circuits in parallel freed up circuits used by critical patients. The final effect was an effective increase in the total circuit area and the consequent decrease in its resistance.

\subsection{Analysis of the bottlenecks}

Eliyahu M. Goldratt was one of the developers of the Theory of Constraints. ${ }^{[16]}$ He compared the stages of a complex process with the stages of a chemical reaction, in which the velocity of the slower stage determines the velocity of the entire process. ${ }^{[17]}$ One of the most important restrictions in mass transportation (as in the case of chemical reactions) is the conservation of matter. In mathematical terms, flow $\phi$ is written as Equation 5:

$$
\phi=\rho v A
$$

This is the Continuity Equation. Here $\rho$ is the density of the material that flows, $v$ its velocity and $A$ is the area of the channel's transversal section. Therefore, matter is conserved and it results in the following equation (see Equation 6):

$$
\phi=\rho_{1} v_{1} A_{1}=\rho_{2} v_{2} A_{2}=\ldots=\rho_{n} v_{n} A_{n}
$$

valid for any segment $1,2, \ldots, n$, of the circuit. If density $\rho$ is constant, since the material that flows is the same, it will be the following condition (see Equation 7):

$$
v_{1} A_{1}=v_{2} A_{2}=\ldots=v_{n} A_{n}
$$

From Equation 7 it follows that the flow does not depend only on the velocity for being constant. There is an inverse relation between the velocity of the fluid in a channel and the area of its transversal section. If this area is decreased (a bottleneck), the velocity of the fluid has to increase in the same proportion to keep the flow constant. If this pro- 
portional increase in velocity is not observed, there will be an overflow of the fluid through the channel's sides. When there is no room for such overflowing, there is retention of the fluid and this effect results in return pressure, generating turbulence and dissipative effects (friction) in the flow. The result is the increase in the pressure and the buildup of fluid in the entrance of the system. In the treatment of patients in a local in-series circuit, the base $b(N)$ and area A are minimal. That is why the velocity of the flow in this conductor must be increased in order to keep the value of flow $\phi$ constant. If its velocity decreases, there will be a drop of flow, with the subsequent appearance of a bottleneck. That is why some circuits with $N=1$, especially in series, deserves special attention from the manager, in order to avoid being transformed into bottlenecks.

The goal to fix a bottleneck demands the increase in either velocity of the flow or transversal section area. The increase in velocity equally implies: an increase in the organization and the quality of the attendance in a well-done triage, standardized work sequences, the minimization of waste and flaws, the mapping and shortening of processes, the reduction of variability and pulling, instead of pushing the patients throughout the healthcare delivery. ${ }^{[5]}$ All of these measures reduce the friction, the length and the time, and increase the velocity of the flow.

The adjustment of the capacity to the demand, such as, for instance, the reallocation of professionals in periods of more demand, creates more attendance channels. Besides, it is possible to combine different steps and processes for them to act simultaneously, as long as they are compatible among themselves. All these decisions can generate conductors in parallel. Prioritizing such configurations increase the area of the transversal section, regardless of the process being technical or administrative. The parallelism for the patient flow was studied in other models, such as the Systems Thinking ${ }^{[6,9]}$ and the Queuing Theory. ${ }^{[12,21]}$

\subsection{Analysis of the attendance circuits in an emergency department}

Considering the diverse aspects of the proposed analogy in this work, we can presume the existence of three basic types of circuits applied to the emergency department:

a) Type 1: Short go home circuit. In this type of circuit, the patient mainly goes through the line of medical attendance and is discharged to go home. This type of circuit meets the classes called Fast Track, Super Track or Ultra Track. ${ }^{[9]}$ The patients that go through this circuit present low complexity in the medical demand. That is why this circuit offers less resistance to the patient flow. Its main characteristics are:
(1) Its length $L$ is shorter, given that medical offices are usually located close to the circuit's entry and exit.

(2) The organization of the lines is simple and depends only on the service of triage and reception (used to make the treatment file) and on the medical treatment. By its nature, this circuit has a low internal friction $\mu$.

(3) There are more available medical offices that characterize the lines $b(N)$. This condition increases in an easily realizable manner, the area $A$. In this circuit, the treatment lines are found almost always in parallel.

(4) This circuit supports more pressure from patients in the entry point, and the pressure in the exit is zero since the patient goes home after being discharged by the physician.

These characteristics guarantee that this circuit admits high values (volume) of flow and rarely interrupts the attendance (its saturation rarely occurs).

b) Type 2: Long go home circuit. In this circuit, the patient goes through lines that have already been described for circuit type 1 (reception, triage and medical treatment). Besides these lines, they are sent to other circuits that include other physicians, nurses, the administration of drugs, laboratory and image examinations. After this process, longer than the previous one, the patient is discharged to go home. The main characteristics of this circuit type are:

(1) Length L longer than the previous one and the demand for architecture projects that prioritize treatment in the same physical level. Additional local circuits, such as image diagnostic equipment close to the treatment line, are actions that can shorten its value.

(2) Internal friction $\mu$ is higher than that of the previous type. There are more conductors involved, the connection between the conductive elements is more complex, and the quality and the organization become more important.

(3) Area A decreases due to a larger occupation of the lines. In this type there are more intermediate circuits and the configuration in series becomes more important.

(4) Pressure difference still depends mainly on the pressure at the entry, since the pressure at the exit could still be small, because the patient will go home, as long as the discharge is given at an adequate time.

By its characteristics, this circuit presents a higher possibility of having its attendance interrupted, and reaching saturation, when compared to the type 1 circuit.

c) Type 3: Long go into the hospital circuit. In this type of circuit, the patient goes through lines of circuit type 1 or 
2 and is sent to treatment and admittance in other circuits inside the hospital.

This circuit has a property that differentiates it from the other two types: the exit. The patient leaves the emergency department to enter another circuit of the hospital. In this case, a saturation of the attendance lines could take place, especially due to the difficulty of having available beds in the patient care unit, intensive care unit or operating rooms. It is common to keep the patient temporarily in the emergency department, an effect known as "boarding". ${ }^{[9]}$ That is why the pressure in the exit of the emergency department increases, as there is equivalent pressure build-up at the entry. As a result, the value of the difference in pressure is neutralized and there would be no flow $\phi$. Consequently, the saturation of the lines takes place, due to patients waiting to be admitted. Thus, the value of $N$ is annulled and resistance $R$ attains very high value. This is the circuit type that interrupts the emergency services the most.

\section{Discussion}

Hospital managers wonder on how to understand the complexity and variability of the processes related to the assistance of patients. In this sense, hospital management is intrinsically interdisciplinary and demands both medical and administrative coordination.

As pointed out by Deming ${ }^{[9]}$ "in order to optimize the system, everyone working in it needs to clearly understand the overall aim of the system. We cannot optimize the entire system by separately optimizing each part of the system". With the same reasoning, Senge ${ }^{[34]}$ argues that "there must be shared mental models that consider the fundamental interconnections of the systems". In this sense, the physical analogy for the patient flow allows the visualization of the system and the development of corrections to several processes involved in it. Most analyses of the management process reinforce the effects of complexity and the regimen of non-linearity of the patient flow, although they intuitively manage the system within the linear regime, a procedure known as "the smooth patient flow". ${ }^{[2,5]}$ However, as pointed out in this work, the non-linear effects occur above $80 \%$ to $85 \%$ of occupation of the installed capacity. As shown here, the analogy of the attendance lines in a hospital with Ohm's Law allows a clear understanding of patient flow, especially in the linear regimen, which occurs in the larger part of the occupation of the installed capacity. The reasoning developed here suggests that the resistance of an attendance circuit can be justified within the context of Little's Law. ${ }^{[35]}$ Little's Law is a linear equation that deals with queuing systems. When it is applied to operations management in an emergency department, Little's Law says that the average number of patients in process equals the average arrival rate of patients multiplied by the average length of stay in the emergency department. ${ }^{[35]}$ It applies to individual queues and also networks. Developments in this direction are in progress in our group.

The model presented here allows a qualitative analysis of the problem and a precise evaluation about how and where the patient flow management tools can be applied. Furthermore, the proposed model helps in the classification and systematization of these tools. This analysis can be made both for part of the circuit as for general circuits, either technical or administrative. Thus, we propose that the attendance circuits are managed in a way that keeps them in the linear regimen, avoiding their saturation instead of their posterior correction. The analogy with the physical phenomena of mass transport supplies the conceptual elements needed to obtain the conditions of linearity.

Ohm's Law, applied in analogy to the patient flow, allows the theory of electrical circuits with its laws and theorems to be employed in the development of software for real time monitoring for the circuit of patients. For that, the use of RFID (radio frequency identification tags) proves to be a promising tool in tracking the patient flow and could be useful in the identification of bottlenecks. ${ }^{[5,33]}$

\section{Conclusions}

The basis for this work is supported on the possibility of using the analogy of physical systems (flow systems) and mass transportation to the problem of management of the patient flow in a healthcare unit. In comparison with this analogy, we used several tools of patient flow management that already existed, showing that the concepts presented in them are consistent with our work. For instance, it was demonstrated how the physical concept of difference of electric potential could be applied to an analogous model of difference in patient pressure in an attendance line. Also it was presented how the physical concepts of resistance and characteristics of an electric conductor could be qualitatively applied to an analogous model capable of describing the patient flow.

Finally, we demonstrated the concepts that allow the manager to make a rational decision and equation for the problem of patient flow. In particular it was verified that, given the evidence of a strong linear regimen in the relation of demandcapacity in a hospital, the use of the analogy presented here could favor the planning of the operation within this linear regimen and eventually quantify, in a preventive manner, the linear deviations. In this way, the manager can correct such deviations in a timely manner, without the need for embarking on expensive corrections in the system when it reaches the non-linear regimen. In these conditions, this work allows 
managers not only to develop a qualitative analysis of the systems in the institutions they manage, but also to formalize the variables customized, aimed at eventually developing quantitative management models that have real impact on the improvement of patient flow.

\section{CONFlicts OF InTEREST Disclosure}

The authors declare they have no conflicts of interest.

\section{REFERENCES}

[1] Fillingham D, Jones B, Pereira P. The challenge and potential of whole system flow: improving the flow of people, information and resources across whole health and social care economies. London: The Health Foundation; Advancing Quality Alliance; 2016 [cited August 4, 2018]. Available from: https://www.health.org.uk/sites/health/files/C hallengeAndPotentialof WholeSystemFlow.pdf

[2] Institute for Healthcare Improvement - IHI. Optimizing patient flow: moving patients smoothly through acute care settings. Innovation Series 2003. Boston, MA; 2003.

[3] Karakusevic S. Evidence for better health care: understanding patient flow in hospitals. London: NuffieldTrust; 2016 [cited August 4, 2018]. Available from: https://www.nuffieldtrust.org.uk /resource/understanding-patient-flow-in-hospitals

[4] The Health Foundation - THF. Improving patient flow: how two trusts focused on flow to improve the quality of care and use available capacity effectively. London: The Health Foundation; 2013 [cited August 5, 2018]. Available from: https://www.health.o rg.uk/publication/improving-patient-flow

[5] Jensen K, Mayer TA, Welch SJ, et al. Leadership for smooth patient flow: improved outcomes, improved service, improved bottom line. Chicago (IL): Health Administration Care; 2007.

[6] Mayer T, Jensen K. Hardwiring flow: systems and processes for seamless patient care. Pensacola (FL): Fire Starter Publishing; 2009.

[7] Litvak E. editor. Managing patient flow: strategies and solutions. 2nd ed. Joint Commission Resources. Gulf Breeze (FL): Fire Starter Publishing; 2010.

[8] Hall R. editor. Patient flow: reducing delay in healthcare delivery. 2nd ed. New York: Springer; 2013. https://doi.org/10.1007/ 978-1-4614-9512-3

[9] Jensen K, Mayer T. The patient flow advantage: how hardwiring hospital-wide flow drives competitive performance. Aché Management Series. Pensacola (FL): Fire Starter Publishing; 2015.

[10] Silva D. Improving patient flow across organizations and pathways. London: The Health Foundation Inspiring Improvement; 2013 [cited August 5, 2018]. Available from: https://www.health.org.uk/publication/improving-p atient-flow-across-organisations-and-pathways

[11] Wiler JL, Griffey RT, Olsen T. Review of modeling approaches for emergency department patient flow and crowding research Acad Emerg Med. 2011 Dec; 18(12): 1371-9. PMid: 22168201 https://doi.org/10.1111/j.1553-2712.2011.01135.x

[12] Bhattacharjee P, Ray PK. Patient flow modeling and performing analysis of healthcare delivery processes in hospitals: a review and reflections. Computers \& Industrial Engineering. 2014; 78: 299-313. https://doi.org/10.1016/j.cie.2014.04.016

[13] Rutherford PA, Provost LP, Kotagal U, et al. Achieving hospitalwide patient flow: the right care, in the right place, at the right time. IHI White Paper, Cambridge, Institute for Health Improvement; 2017 [cited August 5, 2018]. Available from: http://www.ihi.org/resources/Pages/IHIWhitePa pers/Achieving-Hospital-wide-Patient-Flow. aspx

[14] Lima FXC, Belderrain MCN. Propostas de melhorias de atendimento num pronto socorro utilizando teoria de filas e teoria de restrições [Proposals for Attendance Improvement in Emergency Department Using Queuing Theory and Theory of Constraints]. Proceedings of the 13rd ENCITA. São José dos Campos. (SP): ITA. Portuguese. 2007 October 1-4. (Portuguese).

[15] Trevisan MS, Costa CKF, Massuda EM, et al. Teoria das filas: uma aplicação em pronto atendimento de saúde [QueuingTheory: An Application in Emergency Department]. Enciclopédia Biosfera. 2013; 9(19): 2923-2935. (Portuguese).

[16] Goldratt EM. What is this thing called theory of constrains and how should it be implemented? Great Barrington (MA): The North River Press; 1990

[17] Phipps B. Hitting the bottleneck. AGI Goldratt Institute: Milford; 2010.

[18] Sabbadini FS, Gonçalves AA, Oliveira MJF. Gerenciamento de restrições na identificação e solução de problemas em emergência hospitalar: uma abordagem orientada ao paciente [Constraints Management for Identification and Solution of Problems in Emergency Department: a Patient Oriented Approach]. Proceedings of the XIII SIMPEP; 2006a November 6-8. (Portuguese).

[19] Sabbadini FS, Gonçalves AA, Oliveira MJF. The Theory of Constraints and the Simulation Applied in the Capacity Management of Attendance in Emergency Hospital. Revista Produção online. 2006b Dec; 6(3): 51

[20] Womack JP, Byrne AP, Fiume OJ, et al. Going lean in health care. Innovation Series, 2005. Cambridge (MA): Institute for Health Improvement; 2005.

[21] Jiang L, Giachetti RE. A queueing network model to analyze the impact of parallelization of care on patient cycle time. Health Care Manag Sci. 2008 Sep; 11(3): 248-61. https://doi.org/10.100 7/s10729-007-9040-9

[22] Sartor F, Santos J, Airton A, et al. Theory of constraints and simulation applied on demand management of a casualty department. Revista Espacios. 2014; 35(4): 18. Available from: http: //www.revistaespacios.com/a14v35n04/14350419.html

[23] Envision Health Care. Emergency Department Operations Management and Patient Flow: An Envision Playbook - Best Practices, Tools \& Timelines; 2017 [cited 2018 august 5]. Available from: https://www. envisionphysicianservices.com/campaign s/breakthrough-series/presentation-materials/playb ooks/ed-operations-management-and-patient-flow-pla ybook.pdf

[24] Noon CE, Hankins CT, Côté MJ. Understanding the impact of variation in the delivery of healthcare services. J Healthc Manag. 2003 Mar-Apr; 48(2): 82-97, discussion 97-8. PMid: 12698610. https://doi.org/10.1097/00115514-200303000-00004

[25] Gainty C. Mr. Gilbreth's Motion Pictures-The Evolution of Medical Efficiency. N Engl J Med. 2016 Jan 14; 374(2): 109-11. PMid: 26760083. https://doi.org/10.1056/NEJMp1514048 
[26] Hartzband P, Groopman J. Medical Taylorism. N Engl J Med. 2016 Jan 14; 374(2): 106-8. PMid: 26760082. https ://doi .org/10.1 056/NEJMp1512402

[27] Pereira Filho A. PS virou butique de saúde .[The ED became a boutique]. UOL, Economia; 2017 [cited March 20, 2018]. Available from: https://www.uol/economia/especiais/entrevi sta-denise-santos-beneficencia-portuguesa.htm

[28] ONA. National Organization of Accreditation. [cited August 4, 2018]. Available from: https://www.ona.org.br

[29] NIAHO. National Integrated Accreditation for Healthcare Organizations. [cited August 4, 2018]. Available from: https://www.dnvg l.us

[30] JCI. Joint Commission International. [cited August 4, 2018]. Available from: https://www.jointcommissioninternational.o rg
[31] ISO. International Organization for Standardization. [cited August 4, 2018]. Available from: https://www.iso.org

[32] Silva, Roberto Carlos de Oliveira e (Felicio Rocho Hospital, Belo Horizonte, MG). Personal communication. 2018.

[33] Drazen E, Rhoads J. Using tracking tools to improve patient flow in hospitals. California Health Foundation. 2011; 1-21. Available from: https://www. chcf.org/wp-content/uploads/2017/ 12/PDF-UsingPatientTrackingToolsInHospitals .pdf

[34] Senge PM. A quinta disciplina: arte e prática da organização que aprende [The Fifth Discipline: The Art \& Practice of the Learning Organization]. 4th ed. São Paulo: Best Seller; 1999.

[35] Little JDC. Little's Law as Viewed on Its 50th Anniversary. Operations Research. 2011; 59(3): 536-549. https://doi.org/10.128 7/opre.1110.0940 